\title{
Finite temperature QCD coupling constant - imaginary versus real time framework
}

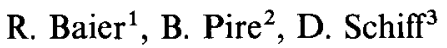 \\ 1 Fakultät für Physik, Universität Bielefeld, W-4800 Bielefeld, Federal Republic of Germany \\ ${ }^{2} \mathrm{CPT}^{\star}$, Ecole Polytechnique, F-91128 Palaiseau Cedex, France \\ ${ }^{3}$ LPTHE ${ }^{\star}$, Université Paris-Sud, Bâtiment 211, F-91405 Orsay, France
}

Received 10 December 1990

\begin{abstract}
The correspondence between the imaginary time and real time thermal running coupling constant is discussed. The temperature dependence is derived in pure QCD at one-loop order in a generalized momentum subtraction (MOM) renormalization scheme.
\end{abstract}

\section{Introduction}

The extension of perturbative field theory methods to finite temperature problems has been developed following two frameworks $[1,2]$. In the so-called imaginary time framework, energies are discretized as

$\omega=2 \pi j T$,

where $j$ is integer or half-integer for bosons and fermions, respectively, and an analytic continuation has to be applied before physical observables are obtained. In the real time formalism [2], one gets directly dynamical quantities but at the price of doubling the degrees of freedom by introducing ghost fields (thereafter denoted as 2-fields in contradistinction to physical external fields denoted as 1-fields).

Green's functions are not simply related to one another in these two frameworks although results for physical observables should be identical. The simplest examples are self-energy and vertex Green's functions. They are of special interest for deriving the behaviour of the running coupling constant at large $T$. Recently, in the real time framework, we studied [3] the one-loop coupling constant, in pure QCD within a generalized momentum subtraction (MOM) renormalization scheme. This scheme, relevant for thermal dynamical processes in which an inherent energy scale is present, generalizes the MOM renormalization procedure [4] to finite $T$. Propagators and vertices are renormalized at space-like

* Laboratoire propre du Centre National de la Recherche Scientifique

$\star \star$ Laboratoire associé du Centre National de la Recherche Scientifique momenta (at typical scale $M$ ) and at a given temperature. We find that the running coupling behaves at fixed $M$ and large $T$ as

$g_{R}^{2}(T) \sim T^{-3}$.

This result, independently confirmed by Nakkagawa et al. [5], extends (and partially corrects) the thermal renormalization group analysis for QCD initiated by Fujimoto et al. [6]. The strong $T$ dependence is due to the occurrence of contributions involving high powers of statistical distributions in the one-loop vertex function and is at variance with the imaginary time results [7].

Indeed, in this last framework, at most one power of the gluon statistical distribution appears in the oneloop vertex, giving rise to an expected behaviour of $g_{R}^{2}(T) \sim T^{-1}$, instead of (2). Is this formalism dependence of the coupling meaningful?

In order to answer this question, it is necessary to study the link between the analytically continued imaginary time two- and three-point Green's functions and the real time Green's functions defined for 1- and 2-fields. The special renormalization scheme studied in [3] is attached to the real time three-gluon vertex $\Gamma_{111}(p, q, r)$. This is not a natural choice to make when comparing with the imaginary time vertex. Instead, one should consider the combination of real time vertices $\Gamma_{a b c}$ obtained through analytic continuation from the imaginary time vertex.

In a recent study, Kobes [8] discusses the correspondence between the imaginary time and the real time formalisms (see also [9]). On the basis of causality arguments he advocates that the Green's functions of interest in the real time formalism are the retarded products of 1 -fields. In the case of the $\lambda \phi^{3}$ theory, he shows that for the self-energy function, the retarded product coincides for positive external energy with the continuation of the imaginary time self-energy function when using the retarded prescription. Actually, for the case of the vertex function, there are several available retarded products depending on the choice of the largest time external leg.

We investigate here the Feynman prescription for the analytic continuation. It is uniquely defined whatever the 
signs of the external energies and it coincides with the retarded prescription when continuing to positive external energy legs.

We then derive the asymptotic behaviour of $g_{\mathbf{R}}^{2}(T)$ in the limit of large $T$, fixed $M$, and we find for pure QCD (in the Feynman gauge) the crucial feature that asymptotic freedom is lost. This raises the question of how to define an improved perturbation theory at large $T$.

Let us now outline the content of the paper. In Sect. II, we first consider the boson self-energy in the case of $\lambda \phi^{3}$, showing in detail the analytic continuation and the relation between the imaginary time and the real time expressions. We then consider the three-boson vertex computation. In Sect. III, we turn to the QCD case and discuss the large $T$ behaviour of the effective coupling constant. Section IV is the conclusion.

\section{Self-energy and vertex in $\lambda \phi^{3}$ field theory}

We study in detail the analytic continuation in order to see explicitly the correspondence between imaginary time and real time results. Let us first consider in the $\lambda \phi^{3}$ model in $\mathrm{D}+1$ dimensions the boson self-energy diagram drawn in Fig. 1. In the imaginary time framework, with Feynman rules taken from [1], one gets

$$
\bar{\Sigma}\left(\omega_{p}, \mathbf{p}\right)=-\lambda^{2} T \sum_{j=-\infty}^{+\infty} \int \frac{\mathrm{d}^{D} k}{(2 \pi)^{D}} \bar{\Delta}(k) \bar{\Delta}(k-p),
$$

where the bare propagator is

$$
\bar{\Delta}(k)=\bar{\Delta}\left(\omega_{k}, \mathbf{k}\right)=\frac{1}{\omega_{k}^{2}+\mathbf{k}^{2}},
$$

and $\omega_{k}=2 \pi j T$, together with $\omega_{p}=2 \pi n T$, and $j, n$ integers. With $|\mathbf{k}|=E_{1}$ and $|\mathbf{p}-\mathbf{k}|=E_{2}$, the frequency sum leads to

$$
\begin{aligned}
\bar{\Sigma}\left(\omega_{p}, \mathbf{p}\right)= & \bar{\Sigma}^{T=0}\left(\omega_{p}, \mathbf{p}\right) \\
& -\frac{\lambda^{2}}{2} \int \frac{\mathrm{d}^{D} k}{(2 \pi)^{D}}\left\{\left[\frac{1}{\left(\omega_{p}+i E_{1}\right)^{2}+E_{2}^{2}}\right.\right. \\
& \left.\left.+\frac{1}{\left(\omega_{p}-i E_{1}\right)^{2}+E_{2}^{2}}\right] \frac{n_{B}\left(E_{1}\right)}{E_{1}}+\left(E_{1} \leftrightarrow E_{2}\right)\right\} .
\end{aligned}
$$

The statistical distribution is $n_{B}\left(E_{1}\right)=\left(\mathrm{e}^{\beta E_{1}}-1\right)^{-1}$, with $\beta=1 / T$. Going back to Minkowski space and real energy $p_{0}$ according to the Feynman prescription for the analytic continuation, $\omega_{p}=-i\left(p_{0}+i \varepsilon p_{0}\right)$, yields

$$
\begin{aligned}
\bar{\Sigma}_{F}\left(p_{0}, \mathbf{p}\right)= & \bar{\Sigma}_{F}^{T=0}\left(p_{0}, \mathbf{p}\right)+\frac{\lambda^{2}}{2} \int \frac{\mathrm{d}^{D} k}{(2 \pi)^{D}} \\
& \cdot\left\{\left[\frac{1}{\left(p_{0}-E_{1}\right)^{2}-E_{2}^{2}+i \varepsilon \operatorname{sign}\left[\left(p_{0}-E_{1}\right) p_{0}\right]}\right]\right. \\
& \left.+\frac{1}{\left(p_{0}+E_{1}\right)^{2}-E_{2}^{2}+i \varepsilon \operatorname{sign}\left[\left(p_{0}+E_{1}\right) p_{0}\right]}\right] \\
& \left.\cdot \frac{n_{B}\left(E_{1}\right)}{E_{1}}+\left(E_{1} \leftrightarrow E_{2}\right)\right\} .
\end{aligned}
$$

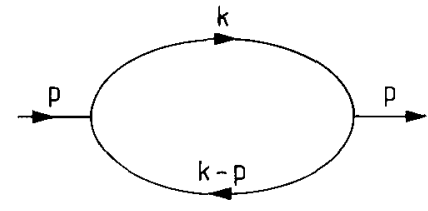

Fig. 1. One-loop scalar self-energy diagram

Using the identiy

$\frac{1}{x \pm i \varepsilon \operatorname{sign}(f)}=\frac{1}{x+i \varepsilon}+2 i \pi \delta(x) \theta(\mp f)$

we get

$$
\begin{aligned}
\bar{\Sigma}_{F}\left(p_{0}, \mathbf{p}\right)= & \bar{\Sigma}_{F}^{T=0}\left(p_{0}, \mathbf{p}\right) \\
& +\lambda^{2} \int \frac{\mathrm{d}^{D+1} k}{(2 \pi)^{D}}\left\{n _ { B } ( | k _ { 0 } | ) \delta ( k ^ { 2 } ) \left(\frac{1}{(p-k)^{2}+i \varepsilon}\right.\right. \\
& \left.+2 i \pi \delta\left((p-k)^{2}\right) \theta\left[\left(k_{0}-p_{0}\right) p_{0}\right]\right) \\
& +n_{B}\left(\left|p_{0}-k_{0}\right|\right) \delta\left((p-k)^{2}\right) \\
& \left.+\left(\frac{1}{k^{2}+i \varepsilon}+2 i \pi \delta\left(k^{2}\right) \theta\left(-k_{0} p_{0}\right)\right)\right\} .
\end{aligned}
$$

In order to make contact with real time, let us briefly recall the Feynman propagators, using the notation of [10]. The free scalar propagator is

$i D_{a b}(p)=U(\beta, p)\left(\begin{array}{cc}\Delta(p) & 0 \\ 0 & \Delta^{*}(p)\end{array}\right) U(\beta, p)$,

where $a, b=1,2$, and

$\Delta(p)=\frac{i}{p^{2}+i \varepsilon}$.

The matrix $U$ is

$U(\beta, p)=\left(\begin{array}{cc}\cosh \theta & \sinh \theta \\ \sinh \theta & \cosh \theta\end{array}\right)$,

and

$\sinh ^{2} \theta=n_{B}\left(\left|p_{0}\right|\right)=\frac{1}{\mathrm{e}^{\beta\left|p_{0}\right|}-1}$,

is the Bose-Einstein statistical factor for the scalar field.

The full propagator $i \hat{D}_{a b}$ has a similar form

$i \hat{D}_{a b}(p)=U(\beta, p)\left(\begin{array}{cc}\hat{\Delta}(p) & 0 \\ 0 & \hat{\Delta}^{*}(p)\end{array}\right) U(\beta, p)$,

which, together with the Schwinger-Dyson equations

$i \hat{D}_{a b}=i D_{a b}+\sum_{c, d=1,2} i D_{a c}\left(-i \Sigma_{c d}\right) i \hat{D}_{d b}$,

leads to write the self-energy matrix $\Sigma$ under the form

$$
-i \Sigma_{a b}(p)=U(\beta, p)^{-1}\left(\begin{array}{cc}
-i \Sigma_{F}(p) & 0 \\
0 & i \Sigma_{F}^{*}(p)
\end{array}\right) U(\beta, p)^{-1},
$$


where the complex function $\Sigma_{F}$ acts as the self-energy for the full scalar propagator

$$
\hat{\Delta}(p)=\frac{i}{p^{2}-\Sigma_{F}+i \varepsilon} .
$$

Let us recall [10] that $\Sigma_{F}$ is a physical quantity, as it determines the pole in the full propagator. In particular its imaginary part is related to the boson damping rate at finite $T$. In contradistinction $\Sigma_{11}$ in this respect is not a physically meaningful object. The relation between $\Sigma_{F}$ and the $\Sigma_{a b}$ is

$$
\Sigma_{F}\left(p_{0}, \mathbf{p}\right)=\Sigma_{11}\left(p_{0}, \mathbf{p}\right)+\mathrm{e}^{-\beta\left|p_{0}\right| / 2} \Sigma_{12}\left(p_{0}, \mathbf{p}\right) \text {. }
$$

It is remarkable that $\Sigma_{F}$ is identical to $\bar{\Sigma}_{F}$ (see also Chapt. 3 of [2]). For $p_{0} \geqq 0, \Sigma_{F}$ coincides with the retarded product of the 1 -fields $\Sigma_{R}$ given by [8],

$\Sigma_{\boldsymbol{R}}\left(p_{0}, \mathbf{p}\right)=\Sigma_{11}\left(p_{0}, \mathbf{p}\right)+\mathrm{e}^{-\beta p_{0} / 2} \Sigma_{12}\left(p_{0}, \mathbf{p}\right)$.

Contrary to $\Sigma_{11}$ and $\Sigma_{12}, \Sigma_{F}$ involves only one power of the statistical distribution $n_{B}$. In the zero-momentum limit, it is free of $\delta^{2}\left(k^{2}\right)$ type singularities, as discussed in [11] for the massive case.

Let us now turn to the case of the one-loop vertex (Fig. 2) which we write in imaginary time as

$$
\begin{aligned}
\bar{\Gamma}= & \lambda^{3} T \sum_{j=-\infty}^{j=+\infty} \int \frac{\mathrm{d}^{D} k}{(2 \pi)^{D}} \\
& \frac{1}{\left(\omega_{k}^{2}+E_{1}^{2}\right)\left(\left(\omega_{k}+\omega_{p}\right)^{2}+E_{2}^{2}\right)\left(\left(\omega_{k}-\omega_{q}\right)^{2}+E_{3}^{2}\right)},
\end{aligned}
$$

with $\omega_{k}=2 \pi j T, \omega_{p}=2 \pi n T, \omega_{q}=2 \pi m T, j, n$ and $m$ integers, and $E_{1}=|\mathbf{k}|, E_{2}=|\mathbf{k}+\mathbf{p}|, E_{3}=|\mathbf{k}-\mathbf{q}|$. As previously, we analytically continue this expression using the Feynman prescription: $\omega_{p} \rightarrow-i\left(p_{0}+i \varepsilon p_{0}\right), \omega_{q} \rightarrow-i\left(q_{0}+i \varepsilon q_{0}\right)$.

In view of our subsequent purpose, we than take the static limit of zero external energies $\left(p_{0}=q_{0}=0\right)$. In this case the analytically continued expression (19) remains real, and we find, in terms of a principal value integral,

$$
\begin{aligned}
\bar{\Gamma}_{F}= & \bar{\Gamma}_{F}^{T=0}+\lambda^{3} \mathbf{P} \int \frac{\mathrm{d}^{D+1} k}{(2 \pi)^{D+1}} \\
& \left\{\frac{2 \pi \delta\left(k^{2}\right) n_{B}\left(\left|k_{0}\right|\right)}{\left[(k+p)^{2}+\mathrm{i} \varepsilon\right]\left[(k-q)^{2}+i \varepsilon\right]}\right. \\
& + \text { cyclic permutations in } k, k+p, k-q\} \\
& +\frac{1}{2} \lambda^{3} \int \frac{\mathrm{d}^{D+1} k}{(2 \pi)^{D+1}}(2 \pi)^{3} \delta\left(k^{2}\right) \delta\left((k+p)^{2}\right) \\
& \cdot \delta\left((k-q)^{2}\right) n_{B}\left(\left|k_{0}\right|\right) .
\end{aligned}
$$

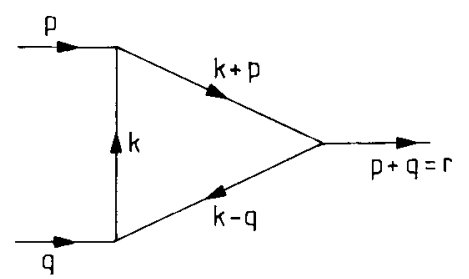

Fig. 2. One-loop scalar vertex diagram
It is now an easy task to show that the above expression is identical (in the static limit) to the following combination of real time vertices,

$$
\begin{aligned}
\Gamma(p, q, r)= & \Gamma_{111}(p, q, r)+\Gamma_{121}(p, q, r) \\
& +\Gamma_{211}(p, q, r)+\Gamma_{221}(p, q, r),
\end{aligned}
$$

with the notation of Fig. 2. For $p_{0}=q_{0}=r_{0}=0$, a helpful intermediate step in going from (21) to (20) is to deduce the following formula

$$
\begin{aligned}
\Gamma= & \Gamma^{T=0}-\lambda^{3} \int \frac{\mathrm{d}^{D+1} k}{(2 \pi)^{D+1}} n_{B}\left(\left|k_{0}\right|\right) \\
& \cdot\left[\Delta(k) \Delta(k+p) \Delta(k-q)+\left(\Delta \leftrightarrow \Delta^{*}\right)\right],
\end{aligned}
$$

using $\Delta(k)+\Delta^{*}(k)=2 \pi \delta\left(k^{2}\right)$.

Three remarks are in order: i) $\Gamma$ coincides with the retarded product of 1-fields [8] (when choosing the leg with momentum $r$ as the largest time leg which with $p_{0}$ and $q_{0}>0$ is a consequence of the causal nature of the retarded product) taken in the static limit; ii) $\Gamma$ involves only one power of $n_{B}$ instead of $n_{B}^{3}$ present in $\Gamma_{111}$; and iii) as for the self-energy there are no $\delta^{2}\left(k^{2}\right)$ or $\delta^{3}\left(k^{2}\right)$ pathologies in the limit of external vanishing momenta [11].

\section{Finite $T$ renormalization and effective coupling in QCD}

We now consider the coupling constant $g_{R}$ in pure QCD renormalized at finite temperature. Since we concentrate on the gluonic sector only, we need the gluon field renormalization constant $Z_{3}$ and the one for the threegluon vertex, $Z_{1}$, in order to calculate

$g_{R}=\frac{Z_{3}^{3 / 2}}{Z_{1}} g$,

in terms of the bare coupling $g$. For our purpose we focus on the one-loop expressions for the gluon self-energy and for the three-gluon vertex. The correspondence between the imaginary and real time frameworks as described in the previous chapter is readily extended to QCD, since obviously the tensor structures are factorized.

As already discussed in the Introduction and described in [3] the renormalization is performed in the generalized momentum (MOM) subtraction scheme at finite $T$. The renormalization constant $Z_{1}$ is defined for the generalization to QCD of the vertex $\Gamma(p, q, r)$ given by (21). Details are illustrated in Fig. 3, and the static space-like configuration $p_{0}=q_{0}=r_{0}=0, p^{2}=q^{2}=r^{2}=$ $-M^{2}$ is considered. The renormalization constant $Z_{3}$ renormalizes the transverse part of the gluon self-energy at $p_{0}=0, p^{2}=-M^{2}$. Throughout we apply the Feynman gauge. Since $\operatorname{Re} \bar{\Sigma}_{F}$ and $\operatorname{Re} \Sigma_{11}$ are the same (cf. (8) and (17)) the constant $Z_{3}$ may be taken from [6],

$Z_{3}=Z_{3}^{T=0}-\frac{g^{2} N}{(4 \pi)^{2}}\left[\frac{4 \pi^{2}}{3 a^{2}}-3 F_{0}(a)-F_{2}(a)\right]+O\left(g^{4}\right)$,

where $N$ is the number of colours. The temperature dependence $T$ enters via $a=\beta M$. However, $Z_{1}$ depends 

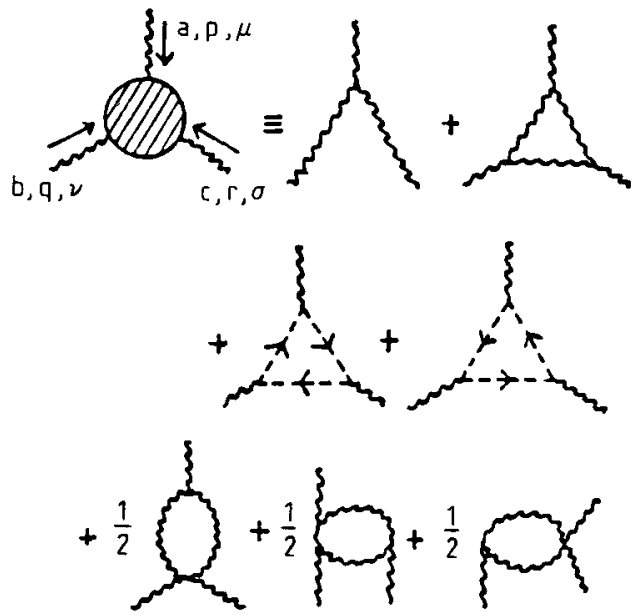

Fig. 3. The thermal three-gluon vertex up to one-loop. The wavy (dotted) lines represent the gluon (ghost)

on the formalism used, since $\Gamma(21)$ and $\Gamma_{111}$ are different. We now obtain

$$
\begin{aligned}
Z_{1}= & Z_{1}^{T=0}-\frac{g^{2} N}{(4 \pi)^{2}}\left[-\frac{2}{3} F_{0}(a)+\frac{7}{3} G_{0}(a)-16 G_{2}(a)\right. \\
& \left.+\frac{\pi}{\sqrt{3}}\left(h_{1}\left(\frac{a}{\sqrt{3}}\right)+\frac{32}{3 a^{2}} h_{3}\left(\frac{a}{\sqrt{3}}\right)\right)\right]+O\left(g^{4}\right) .
\end{aligned}
$$

The functions $F_{0,2}$ and $G_{0,2}$ are already introduced in [6]. The properties of the functions

$h_{m}(y)=\frac{1}{\Gamma(m)} \int_{0}^{\infty} \frac{x^{m-1} \mathrm{~d} x}{\sqrt{x^{2}+y^{2}}} \frac{1}{\left[\exp \sqrt{x^{2}+y^{2}}-1\right]}$,

are studied in [12], in particular their high $T$ expansion.

After applying the same steps as described in $[3,5,6]$ with renormalization group equations at finite $T$ we obtain the running gluon coupling constant as,

$$
g_{\mathrm{R}}^{2}(T)=\frac{g_{R}^{2}\left(T_{0}\right)}{1+\frac{2 N}{(4 \pi)^{2}} g_{R}^{2}\left(T_{0}\right)\left[\Omega_{\text {gluon }}(M / T)-\Omega_{\text {gluon }}\left(M / T_{0}\right)\right]},
$$

with

$$
\begin{aligned}
\Omega_{\text {gluon }}(a=M / T)= & \frac{2 \pi^{2}}{a^{2}}-\frac{23}{6} F_{0}(a) \\
& -\frac{3}{2} F_{2}(a)-\frac{7}{3} G_{0}(a)+16 G_{2}(a) \\
& -\frac{\pi}{\sqrt{3}}\left(h_{1}\left(\frac{a}{\sqrt{3}}\right)+\frac{32}{3 a^{2}} h_{3}\left(\frac{a}{\sqrt{3}}\right)\right) .
\end{aligned}
$$

From the high temperature expansion we find in the high $T$ regime (at fixed $M$ ),

$\Omega_{\text {gluon }}(a=\beta M)=\underset{\substack{a \rightarrow 0 \\ T \rightarrow \infty}}{ }-\frac{31}{9} \frac{\pi^{2}}{a}-\frac{11}{3} \ln a+$ const.
It is convenient to define the thermal $\beta_{T}$-function [13],

$\beta_{T}=T \frac{\mathrm{d} g_{R}^{2}(T, M) / 4 \pi^{2}}{\mathrm{~d} T}$.

At one-loop order it becomes

$\beta_{T}=-c(T)\left(\frac{g_{R}^{2}}{4 \pi^{2}}\right)^{2}$

with $c(T)=(N / 2) T \mathrm{~d} \Omega_{\text {gluon }}(M / T) / \mathrm{d} T$.

Considering again the limit of large $T$, fixed $M$, the thermal coefficient - in this generalized MOM scheme behaves as,

$c(T)=N\left(-\frac{31 \pi^{2}}{18}(T / M)+\frac{11}{6}+\cdots\right)$.

We note that in this limit the thermal one-loop $\beta_{T^{-}}$ function is positive, and no longer leads to asymptotic freedom. The same situation is encountered by Landsman [14], when discussing a manifestly gauge independent QCD coupling in the imaginary time framework. For comparison let us quote his result,

$c(T)=N\left(-\frac{21 \pi^{2}}{18}(T / M)+\frac{11}{6}+\cdots\right)$.

Both coefficients differ from the real time result derived in [3], i.e.

$c(T) \approx+\frac{25 \pi^{2} N}{4}(T / M)^{3}$.

\section{Conclusion}

Based on the correspondence between the imaginary time and real gluon self-energy and three-gluon vertex expressions we calculate the renormalized QCD coupling constant at one-loop order in a generalized MOM subtraction scheme at finite temperature.

This investigation shows that choosing an appropriate combination of three-gluon vertex functions at one-loop in the real time formalism allows to derive the same answer in both real and imaginary time frameworks. In this case, however, the asymptotic freedom property is lost, which indicates that this renormalization prescription is not adequate to improve the perturbative expansion at high temperature.

The specific powers of $(T / M)$ which are present in the large $T$ behaviour of $g_{R}(T)$ are due to the static modes [15], as it is transparent in the imaginary time formalism. It seems that these special powers are not correctly resummed in the thermal renormalization group approach which we deal with in this paper.

As previous observations (see also $[16,17]$ ) already indicate there is in fact no unique way to define a temperature dependent coupling in thermal QCD. It remains to find reasonable prescriptions for physical quantities themselves in order to determine their high temperature behaviour. 
Acknowledgement. Partial support of this work by "Projets de Cooperation et d'Echange" (PROCOPE) is gratefully acknowledged.

\section{References}

1. J.I. Kapusta: Finite-temperature field theory, Cambridge: Cambridge University Press 1989

2. For a review: N.P. Landsman, Ch.G. van Weert: Phys. Rep. 145 (1987) 141

3. R. Baier, B. Pire, D. Schiff: Phys. Lett. B238 (1990) 367

4. W. Celmaster, R.J. Gonsalves: Phys. Rev. D20 (1979) 1420

5. H. Nakkagawa, A. Niégawa, H. Yokota: Phys. Lett. B244 (1990) 63

6. Y. Fujimoto, H. Yamada: Phys. Lett. B195 (1987) 231; B200 (1988) 167; H. Nakkagawa, A. Niégawa, H. Yokota: Phys. Rev. D38 (1988) 2566

7. E. Braaten, R.D. Pisarski: Nucl. Phys. B337 (1990) 569; and references therein
8. R.L. Kobes: Phys. Rev. D42 (1990) 562; Phys. Rev. D43 (1991) 1269

9. A.A. Abrikosov, L.P. Gor'kov, I.E. Dzyaloshinski1: Sov. Phys. JETP 9 (1959) 636; I.E. Dzyaloshinskil: Sov. Phys. JETP 15 (1962) 778; T.S. Evans: Phys. Lett. B249 (1990) 286; Phys. Lett. B252 (1990) 108

10. R.L. Kobes, G.W. Semenoff: Nucl. Phys. B260 (1985) 714; Nucl. Phys. B272 (1986) 329

11. Y. Fujimoto, R. Grigjanis: Z. Phys. C-Particles and Fields 28 (1985) 395

12. H.E. Haber, H.A. Weldon: J. Math. Phys. 23 (1982) 1852

13. H. Matsumoto, Y. Nakano, H. Umezawa: Phys. Rev. D29 (1984) 1116; N.P. Landsman: Comm. Math. Phys. 125 (1989) 643

14. N.P. Landsman: Phys. Lett. B232 (1989) 240

15. A.D. Linde: Phys. Lett. B96 (1980) 289

16. K. Enqvist, K. Kajantie: Mod. Phys. Lett. A2 (1987) 479

17. S. Nadkarni: Phys. Lett. B232 (1989) 362 